\title{
The online Cough Clinic: developing guideline-based diagnosis and advice
}

\author{
P.W. Dettmar*, V. Strugala*, H. Fathi ${ }^{\#}$, H.J. Dettmar*, C. Wright ${ }^{\#}$ and A.H. Morice ${ }^{\#}$
}

\section{ABSTRACT: The aim of the present study was make chronic cough guidelines more practical and user friendly by developing an internet-based interactive diagnostic questionnaire for chronic cough.}

A prospective cohort study of chronic cough sufferers was conducted in the UK, following European Respiratory Society guidelines for the diagnosis and management of chronic cough. Depending on the response to 16 specific questions, the medical condition responsible for the patient's chronic cough was ascertained according to a predetermined diagnostic algorithm designed to differentiate the three common causes of chronic cough. Appropriate advice and treatment recommendations were then provided.

8,546 adults with chronic cough completed the Cough Clinic diagnostic questionnaire. $46.1 \%$ were suggested to have reflux, $38.7 \%$ asthma and $15.2 \%$ rhinitis. Participants found the website easy to use (94\%), the advice helpful $(73 \%)$ and that it helped them to communicate with their general practitioner better $(60 \%)$, and $62 \%$ reported taking the recommended treatment.

The Cough Clinic, an internet-based diagnostic site for chronic cough, had a large uptake by chronic cough sufferers in the UK. Almost half were diagnosed as having reflux as the probable cause of their chronic cough. Internet diagnosis by expert algorithm provides a novel mechanism for patients to access guideline-recommended therapies and enhances dialogue between patients and physicians.

KEYWORDS: Chronic cough, diagnosis, gastro-oesophageal reflux, questionnaire

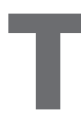

he challenge of making an accurate diagnosis remains one of the most fulfilling roles of physicians [1]. Clinical practice guidelines are considered as important tools to guide evidence-based decision making [2]. However, the routine implementation of guidelines, many of which can be of hundreds of pages, has been reported to be low [3]. Doctors and patients are becoming more proficient in using the internet, with 31.8 million adults in the UK estimated to have internet access (15.2 million households), with searching for health information at the top of the list of reasons for using the web [4]. Google ${ }^{\circledR}$ searchers can find the correct diagnosis for their medical condition in $58 \%$ of cases [5]. Hence, the hypothesis is that a guideline-based web clinic would be a practical way to address patients' healthcare queries.

Chronic cough is defined as a cough lasting for $>8$ weeks and is a disabling symptom with a significant impact on a patient's quality of life. Cough is the commonest symptom for which medical care is sought [6]. In the absence of any obvious respiratory disease, three common causes have been proposed for chronic cough; reflux disease, an asthma syndrome and rhinitis. The European Respiratory Society (ERS) guidelines illustrate the development of the approaches to cough $[7,8]$.

The Cough Clinic (www.coughclinic.org.uk; National Health Service/University of Hull, Hull, UK) is an internet-based diagnostic site developed to suggest a probable diagnosis of the condition causing a patient's chronic cough. The Cough Clinic individualises advice based upon the guidelines set by the ERS [7] thus making the guidelines more practical, while at the same time providing information about the condition for both patients and their physicians.

The diagnosis of the aetiology of cough in a patient requires relevant information from the patient's medical history followed by the assigning of weighting factors to the information to predict the most likely diagnosis. Correct diagnosis is
AFFILIATIONS

*Technostics Ltd, The Deep Business Centre, Hull, and

${ }^{\#}$ Cardiovascular and Respiratory Studies, Hull York Medical School, Castle Hill Hospital, Cottingham, UK

CORRESPONDENCE

A.H. Morice

Hull York Medical School

University of Hull

Castle Hill Hospital

Castle Road

Cottingham

HU16 5JQ

UK

E-mail: a.h.morice@hull.ac.uk

Received:

Aug 182008

Accepted after revision:

March 172009

First published online:

March 262009 
crucial for an effective therapy. An online internet-based system that claims to be able to give an accurate diagnosis should be able to perform all of the above functions.

Here we describe the Cough Clinic website and its role in diagnosis of chronic cough and patient appreciation of the pathway.

\section{METHODS}

\section{Data collection}

On entering the Cough Clinic website the patient was registered, provided consent and demographic data was collected (age, sex and region of residence). Before proceeding with the questionnaire, the patient was required to confirm that a normal chest radiograph had been obtained, as it is mandatory for the investigation of chronic cough.

Ethical approval for the study was granted by the local research ethics committee for Hull and East Riding, UK. Research and development approval was granted by Hull National Health Service Trust, Hull, UK. All patients provided online consent at the time of completion of the Cough Clinic questionnaire.

The questionnaire was set over three pages. The patient was asked about the length of time that they have suffered with cough, and a Likert scale (0-10) used to score the severity of the cough. The patient was asked to answer a series of questions to determine other relevant factors. The second page gathered further information on the patient's smoking habits and medication use. The third page asked about key diagnostic symptoms and their severity by Likert scale ( $0-5)$ (table 1 ). For the diagnosis of reflux-induced cough, these questions were based on the validated reflux symptom index (RSI) [9] and the Hull reflux cough questionnaire (HRCQ) [10]. Asthma symptoms were based on Royal College of Physicians questionnaire [11].
Certain symptoms (e.g. haemoptysis) were identified as "red flag" symptoms and the completion of the questionnaire was terminated with advice to obtain specialist help. Red flag symptoms were only seen in eight patients (three coughing up blood and five coughing up more than one cupful of phlegm).

\section{Algorithm and diagnosis}

An algorithm was applied to the questionnaire responses, after assigning weighting factors, to calculate a score as a percentage of the maximum probability of the three main causes of chronic cough. The condition with the highest percentage was chosen as the most probable diagnosis (reflux, asthma or rhinitis) causing the chronic cough. The algorithm was scored as a percentage of the total possible score for each symptom. Thus, although there were more questions pertaining to reflux the algorithm was not biased towards a reflux diagnosis since each individual question contributed a smaller percentage to the total.

\section{Treatment}

When a diagnosis is made, the ERS guidelines recommend a therapeutic trial of the most appropriate treatment. The Cough Clinic generated a generic letter for the patient to take to their primary care physician that referred to the completed questionnaire, the ERS guidelines and the suggested diagnosis of the cause of the patient's chronic cough; it also suggested the most suitable treatment trial for the patient (see online supplementary material).

\section{Follow-up}

As for any other medical consultation, the clinician needs to assess the effectiveness of the diagnosis and the recommended treatment. After 2 months the patient received a request to complete a follow-up questionnaire over the internet. This allowed for the assessment of the patient's current symptoms, current medications, patient compliance and the efficacy of the

TABLE 1 Questions used to ascertain the probable medical condition responsible for a patient's chronic cough

\begin{tabular}{lll} 
Number & & \multicolumn{1}{c}{ Question } \\
\hline 1 & Cough with eating (during or straight after meals) & \\
2 & Cough with certain foods & Reflux \\
3 & Cough when you get out of bed in the morning & Reflux \\
4 & Cough brought on by singing or speaking (for example, on the telephone) & Reflux \\
5 & Hoarseness or a problem with your voice & Reflux \\
6 & Clearing your throat & Reflux \\
7 & Cough after lying down & Reflux \\
8 & Heartburn, chest pain, indigestion or stomach acid coming up & Asthma \\
9 & Wheezing or chest tightness in general & Asthma \\
10 & Cough waking you from sleep & Rhinitis \\
11 & Shortness of breath when not coughing & Rhinitis \\
12 & Blocked or stuffy nose & Rhinitis \\
13 & Excess mucus in the throat, or dripping down the back of the nose & Rhinitis \\
14 & Itchy nose and/or sneezing & \\
15 & Loss of the sense of smell &
\end{tabular}

The severity of the above key diagnostic symptoms was rated on a Likert scale (0-5). The questions were asked in a random order and not as listed. 
recommended treatment. We also inquired into the patients' satisfaction with the Cough Clinic.

\section{RESULTS}

\section{Demographics}

Between the time the site went live (January 2006) and October $2007,13,610$ people had registered at the site, of which 8,546 $(63 \%)$ patients in the UK completed the Cough Clinic online cough diagnosis questionnaire. There was a female dominance $(57 \%)$ and the mean \pm SD age was $45.5 \pm 16.4 \mathrm{yrs}$, with ages ranging from 18-86 yrs (fig. 1).

The duration of cough, for all of the responders, was $>3$ months; in $64.8 \%$ of patients it was $<1 \mathrm{yr}$ and $13.7 \%$ of patients had had their cough for $>5 \mathrm{yrs}$.

Patients were asked to score the severity of their cough on a Likert scale of $0-10$ (10 being the most severe and frequent cough) and the mean \pm SD score for cough among the patients was $5.85 \pm 2.1$.

The mean $\pm \mathrm{SD}$ cough score varied depending on their suggested diagnosis $(p<0.0001$, one-way ANOVA), with those patients diagnosed with reflux scoring $5.4 \pm 2.2$, those diagnosed with rhinitis scoring $5.8 \pm 2.08$, and those diagnosed with asthma scoring $6.4 \pm 2.15$.

$41.4 \%$ of patients were either current smokers or had previously been smokers. $11 \%$ of responders had previous diagnosis of respiratory illnesses, such as chronic obstructive pulmonary disease (COPD), bronchiectasis, emphysema and asthma, with asthma the greatest (9\% of all).

From the drug history data, $21.6 \%$ of patients were on inhalers, of which $11 \%$ were steroid inhalers. $19 \%$ took blood pressure tablets, with $7 \%$ of these on angiotensin converting enzyme (ACE) inhibitors. The known side-effects of ACE inhibitors include chronic cough, which occurs at the rate of $\sim 15 \%$, depending on the population studied [12].

\section{Specific questions to aid diagnosis}

There were 16 questions that were used to specifically ascertain the medical condition responsible for the patient's chronic

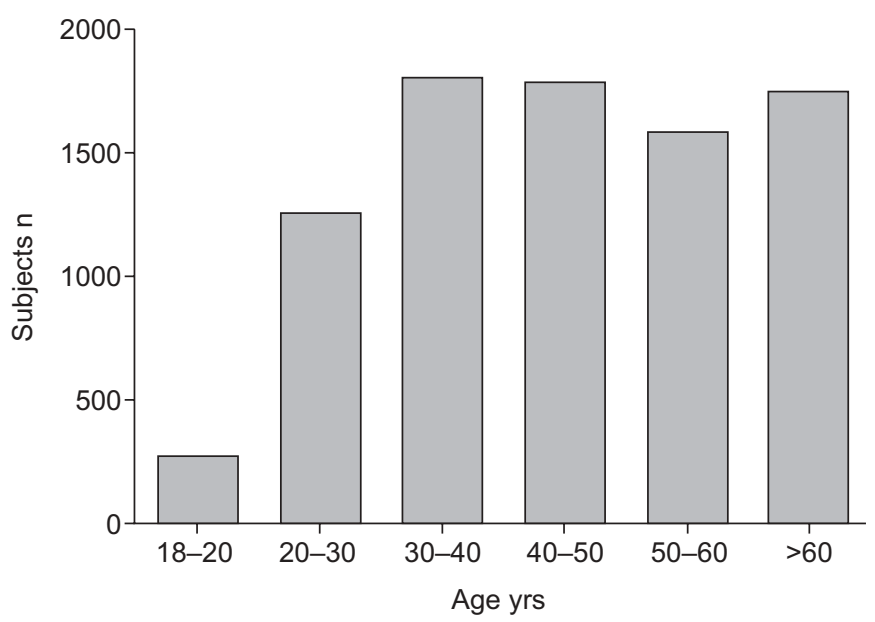

FIGURE 1. Breakdown of ages of the population completing the questionnaire. There was no specific dominant age group. cough (table 1). Each question was scored for severity using a Likert scale of $0-5$.

Depending on the score given for each question, a weighting was applied relevant to the importance of that symptom for the three common causes of chronic cough.

The breakdown of any symptoms reported (score 1-5) by the population group studied is shown in figure 2; it was possible to identify differences between the groups. There was a clear distinction in the type of symptoms reported by those diagnosed with probable asthma and those diagnosed with probable rhinitis. Focusing on the moderate to severe symptoms (score 3-5), which had the weighting factors applied to them, shows that each predicted diagnosis presents with a different profile (fig. 3). Those with probable rhinitis responded highly to questions $12-16$, those with probable asthma responded to questions 9-11, whereas those with probable reflux were those that did not respond high to questions 9-16 but had a high proportion of symptoms related to questions 1-8.

Coughing after lying down was reported to be the most severe accompanying symptom ( $20 \%$ scored it as 5 and $18.5 \%$ as 4 ), while hoarseness was the mildest accompanying symptom (15.8\% scored it as 1 and $17.4 \%$ as 2$)$. Clearing the throat in probable reflux patients, coughing after lying down in probable asthmatic patients, and post-nasal drip in probable rhinitis were the most troublesome symptoms in the different groups.

\section{Validation study}

The Cough Clinic algorithm was tested in a pilot study of 30 patients attending the Hull cough clinic (Hull, UK). Patients were administered the questionnaire before being seen as new patients by the medical team. There was a close association between the web-based Cough Clinic diagnosis and that of the clinician's full work up. Only two patients did not have agreement in diagnosis between the two methods. We recognise that there is insufficient power to provide an accurate estimate of diagnostic accuracy, but took the view that there would be variation between different clinicians similar, if not greater than, that between the algorithm.

\section{Final diagnosis}

Three probable diagnoses, reflux, asthma and rhinitis, were the main outcomes of the questionnaire; 3,936 (95\% CI 3,846-4,027) $(46.1 \%)$ of patients were diagnosed with reflux, 3,310 $(95 \% \mathrm{CI}$ $3,222-3,398)(38.7 \%)$ were diagnosed with asthma and 1,300 (95\% CI 1,173-1,427) (15.2\%) were diagnosed as having rhinitis (fig. 4).

\section{Follow-up questionaire}

A request to complete a follow-up questionnaire 2 months after the initial diagnosis was sent to 8,434 patients. Response was only given by $1,047(12.4 \%)$. Of the patients completing the follow-up questionnaire, $94 \%$ said they found the site easy to use, $73 \%$ found the advice helpful, while $60 \%$ stated that it helped them communicate with their general practitioner (GP) better. $62 \%$ of patients had taken a recommended treatment.

\section{DISCUSSION}

Guidelines and approved evidence-based clinical algorithms are vast swathes of information that are hard to memorise and 


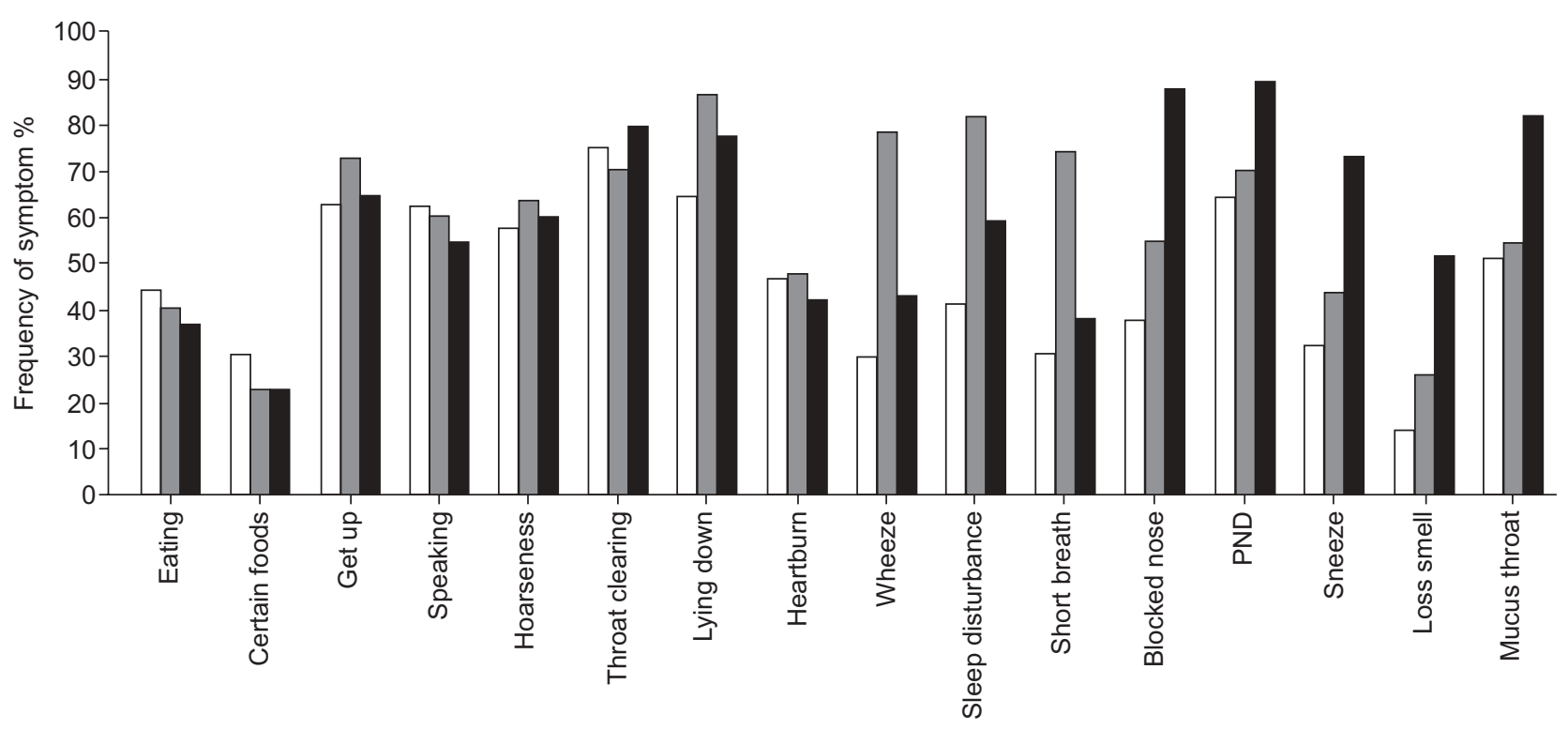

FIGURE 2. Proportion of patients reporting the presence of symptoms from questions 1-16 (score 1-5). $\square$ : reflux; ㅍ: : asthma; $\mathbf{\square}$ : rhinitis. PND: post-nasal drip.

put into practice. The use of online tools to put guidelines into practice and provide quality information to patients is a valuable addition to the healthcare armoury. The use of the Cough Clinic, an internet-based diagnostic website to diagnose the condition causing a patient's chronic cough, was assessed in the current study.

It should be stressed that definite diagnosis cannot be obtained using this internet-based approach without full investigative work-up. The suggested diagnosis is provided with appropriate advice and treatment but further medical consultation is advocated, with a computer-generated letter provided. However, it is more likely to propose a diagnosis that is unlikely to have been put forward by the GP in an initial consultation.
Our previous experience indicates that in chronic cough patients seeking a consultation the overwhelming majority have seen at least one and often two doctors [13].

There has been large utilisation of the Cough Clinic site by adult chronic cough sufferers in the UK covering a wide demographic, with the majority suffering from cough for 3 months to $1 \mathrm{yr}$. The 16-part symptom questionnaire and associated algorithm was the main diagnostic device to determine if reflux, rhinitis or asthma was the probable cause of the chronic cough. The questions were derived from studies characterising the symptom complex associated with each condition. In reflux, our previously determined symptom profile of patients with $\mathrm{pH}$-proven acid reflux and chronic cough was utilised [14]. For asthma, the Royal

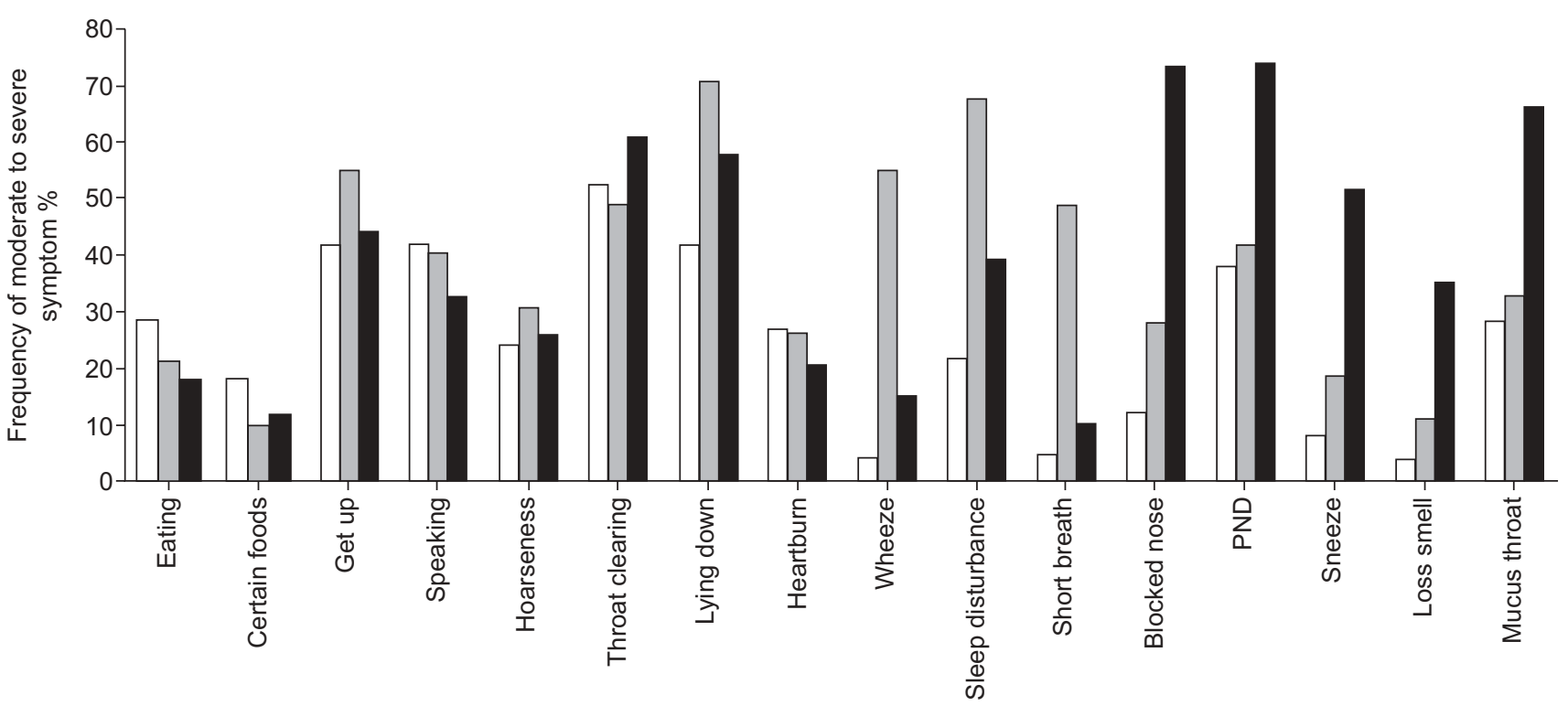

FIGURE 3. Proportion of patients reporting the presence of symptoms from questions 1-16 (score 3-5). $\square$ : reflux; $\square$ : asthma; 


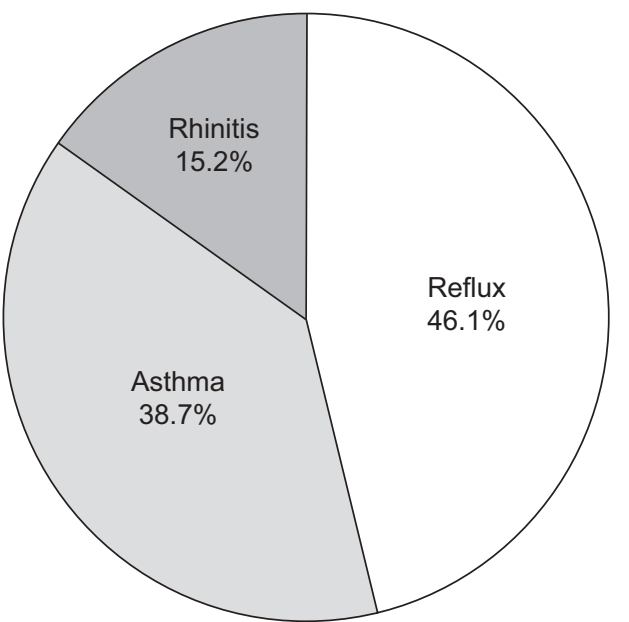

FIGURE 4. Probable diagnosis of medical condition responsible for chronic cough in 8,546 patients completing the Cough Clinic questionnaire.

College of Physicians questionnaire was used [11]. For rhinitis, questions from the rhinosinusitis-specific Sino-nasal Assessment Questionnaire was used [15]. It should be noted that, although the three questionnaire components of the Cough Clinic are validated, the whole questionnaire and algorithm is not. There were three distinct patterns of response to the 16 questions. A high score to questions 9-11 pointed to asthma, high scores in questions 12-16 indicated rhinitis, while consistently moderate to high scores to questions 1-8 signified reflux.

The population of chronic cough sufferers who completed the Cough Clinic process is the largest cohort studied to date by an order of magnitude. Previously published epidemiological studies of chronic cough have used cohorts ranging from 43228 patients, owing to the specialist nature of the condition and that the prevalence data of the three main causes of chronic cough varies widely [16-28]. The prevalence of asthma ranged $6-59 \%$, gastro-oesophageal reflux disease (GORD) $0-41 \%$ and rhinitis $8-56 \%$. We amalgamated the data from these 13 studies, and the overall number of patients was 1,$258 ; 25 \%$ with asthma, $20 \%$ with GORD and $34 \%$ with rhinitis [8].

In the study presented here the prevalence of probable GORD was $46.1 \%$, higher than previously observed, indicating that those presenting to secondary care may be the "tip of the iceberg" and that the condition may be more common than previously believed. Probable asthma was responsible for chronic cough in $38.7 \%$ of our study group population, which is in the range previously seen. Probable rhinitis was less prevalent in chronic cough sufferers than previously found (15.2\%). Our population is significantly different from those previously reported in the literature in that we have not excluded patients with significant airflow limitation. It is possible that other diagnoses, such as COPD, may be responsible for cough in a proportion of the patients reported here; however, it is also possible that patients with airflow obstruction may have one of the underlying causes of cough discussed previously. This is one of the unavoidable limitations of an internet-based algorithm.

The study has given us an interesting insight into the likely aetiology of chronic cough in this biased segment of the general population (internet users seeking medical advice online). In addition, it is our intention to follow-up the patients who have received an initial diagnosis for their chronic cough with a second internet-based questionnaire. This follow-up questionnaire will be used to obtain information on treatment efficacy and will enable us to carry out internet-based clinical trials as well as epidemiological and market research studies. However, to date, follow-up response has been low $(12.4 \%)$; therefore, data gathered thus far has not been presented here.

Although the current study was based only on chronic cough sufferers in the UK (with internet access) it is our intention to extend the internet-based questionnaire to other countries where guidelines are available. This will make it possible to better study the aetiology and the epidemiology of chronic cough across countries and populations.

\section{STATEMENT OF INTEREST}

Statements of interest for P.W. Dettmar, V. Strugala, H.J. Dettmar and A.H. Morice can be found at www.erj.ersjournals.com/misc/statements. dtl

\section{REFERENCES}

1 Pauker SG, Gorry GA, Kassirer JP, et al. Towards the simulation of clinical cognition. Taking a present illness by computer. Am J Med 1976; 60: 981-996.

2 Field MJ, Lohr KN. Clinical Practice Guidelines: Directions for a New Programme. Washington, National Academy Press, 1990.

3 Woolf SH, Grol R, Hutchinson A, et al. Clinical guidelines: potential benefits, limitations, and harms of clinical guidelines. BMJ 1999; 318: 527-530.

4 Powell J, Clarke A. The WWW of the World Wide Web: Who, What, and Why? J Med Internet Res 2002; 4: e4.

5 Tang $\mathrm{H}, \mathrm{Ng} \mathrm{JH}$. Googling for a diagnosis - use of Google as a diagnostic aid: internet based study. BMJ 2006; 333: 1143-1145.

6 Morice AH. Epidemiology of cough. Pulmon Pharmacol Therap 2002; 15: 253-259.

7 Morice AH, Fontana GA, Belvisi MG, et al. ERS guidelines on the assessment of cough. Eur Respir J 2007; 29: 1256-1276.

8 Morice AH, Fontana GA, Sovijarvi AR, et al. The diagnosis and management of chronic cough. Eur Respir J 2004; 24: 481-492.

9 Belafsky PC, Postma GN, Koufman JA. Validity and reliability of the reflux symptom index (RSI). J Voice 2002; 16: 274-277.

10 Faruqi S, Brook H, Hunter V, et al. Reproducibility and sensitivity of the Hull Reflux Cough Questionnaire. Am J Respir Crit Care Med 2009; 179: A575.

11 Juniper EF, O'Byrne PM, Guyatt GH, et al. Development and validation of a questionnaire to measure asthma control. Eur Respir J 1999; 14: 902-907.

12 Ravid D, Lishner M, Lang R, et al. Angiotensin-concerting enzyme inhibitors and cough: a prospective evaluation in hypertension and in congestive heart failure. J Clin Pharmacol 1994; 34: 11161120.

13 Everett CF, Kastelik JA, Thompson RH, et al. Chronic persistent cough in the community: a questionnaire survey. Cough 2007; 3: 5.

14 Everett $\mathrm{CF}$, Morice AH. Clinical history in gastroesophageal cough. Respir Med 2007; 101: 345-348.

15 Fahmy FF, McCombe A. General versus specific sino-nasal outcome measures. Clin Otolaryngol 2002; 27: 283-284.

16 Brightling CE, Ward R, Goh KL, et al. Eosinophilic bronchitis is an important cause of chronic cough. Am J Respir Crit Care Med 1999; 160: 406-410

17 Hoffstein V. Persistent cough in nonsmoker. Can Respir J 1994; 1: $40-47$. 
18 Irwin RS, Corrao WM, Pratter MR. Chronic persistent cough in the adult: the spectrum and frequency of causes and successful outcome of specific therapy. Am Rev Respir Dis 1981; 123: 413-417.

19 Irwin RS, Curley FJ, French CL. Chronic cough. The spectrum and frequency of causes, key components of the diagnostic evaluation, and outcome of specific therapy. Am Rev Respir Dis 1990; 141: 640-647.

20 Marchesani F, Cecarini L, Pela R, et al. Causes of chronic persistent cough in adult patients: the results of a systematic management protocol. Monaldi Arch Chest Dis 1998; 53: 510-514.

21 McGarvey LP, Heaney LG, Lawson JT, et al. Evaluation and outcome of patients with chronic non-productive cough using a comprehensive diagnostic protocol. Thorax 1998; 53: 738-743.

22 Mello CJ, Irwin RS, Curley FJ. Predictive values of the character, timing, and complications of chronic cough in diagnosing its cause. Arch Intern Med 1996; 156: 997-1003.
23 O'Connell F, Thomas VE, Pride NB, et al. Capsaicin cough sensitivity decreases with successful treatment of chronic cough. Am J Respir Crit Care Med 1994; 150: 374-380.

24 Palombini BC, Villanova CA, Araujo E, et al. A pathogenic triad in chronic cough: asthma, postnasal drip syndrome, and gastroesophageal reflux disease. Chest 1999; 116: 279-284.

25 Poe RH, Harder RV, Israel RH, et al. Chronic persistent cough. Experience in diagnosis and outcome using an anatomic diagnostic protocol. Chest 1989; 95: 723-728.

26 Poe RH, Israel RH, Utell MJ, et al. Chronic cough: bronchoscopy or pulmonary function testing? Am Rev Respir Dis 1982; 126: 160-162.

27 Simpson G. Investigation and management of persistent dry cough. Thorax 1999; 54: 469-470.

28 Smyrnios NA, Irwin RS, Curley FJ. Chronic cough with a history of excessive sputum production. The spectrum and frequency of causes, key components of the diagnostic evaluation, and outcome of specific therapy. Chest 1995; 108: 991-997. 